\title{
"Inverse signaling" of the transmembrane chemokine CXCL16 contributes to proliferative and anti-apoptotic effects in cultured human meningioma cells
}

Kirsten Hattermann ${ }^{1 \dagger}$, Kareen Bartsch ${ }^{2 \dagger}$, Henrike H. Gebhardt ${ }^{1}$, H. Maximilian Mehdorn², Michael Synowitz ${ }^{2}$, Anne Dorothée Schmitt ${ }^{2}$, Rolf Mentlein ${ }^{1 \dagger}$ and Janka Held-Feindt ${ }^{2^{*}+}$

\begin{abstract}
Background: Chemokines and their receptors play a decisive role in tumor progression and metastasis. We recently found a new signaling mechanism in malignant glioma cells mediated by transmembrane chemokines that we termed "inverse signaling". According to this hypothesis, soluble (s)-CXCL16 binds to the surface-expressed transmembrane (tm) -CXCL16, and induces signaling and different biological effects in the stimulated cells, so that the transmembrane ligand itself acts as a receptor for its soluble counterpart. Now, we hypothesized that "inverse signaling" via tm-CXCL16 might also take place in meningiomas, a completely different, benign tumor entity.
\end{abstract}

Methods: We used quantitative reverse-transcription polymerase chain reaction, immunocytochemistry and western blot to detect CXCL16 and CXCR6 in human meningioma cells isolated from 28 human meningiomas. Subsequently, we stimulated cultured human tm-CXCL16-positive, CXCR6-negative meningioma cells with recombinant s-CXCL16 and analyzed binding, signaling and biological effects using RNAi silencing to verify specificity.

Results: In fact, cultured human meningioma cells considerably express CXCL16, but substantially lack CXCR6, the only known CXCL16 receptor. These receptor-negative cells could bind s-CXCL16, and responded to s-CXCL16 application with activation of the intracellular kinases ERK1/2 und Akt. As a consequence, we observed increased proliferation and rescue of apoptosis of cultured meningioma cells. Since binding and signaling were abolished by siRNA silencing, we concluded that tm-CXCL16 specifically acts as a receptor for s-CXCL16 also in human meningioma cells.

Conclusion: These findings underline our recent report on the mechanism of inverse signaling as a broad biological process also observable in more benign tumor cells and contributing to tumor progression.

Keywords: Chemokines, Chemokine receptors, Cellular communication, Meningioma, Inverse signaling

\section{Plain english summary}

Cells communicate by ligands that bind to their respective receptors. Some ligands are transmembrane molecules which means they span through the cell membrane. The part of these ligands at the outer cell surface can be liberated from its membrane stack yielding a soluble ligand that is present outside of the cell and can meet

\footnotetext{
* Correspondence: janka.held-feindt@uksh.de

${ }^{\dagger}$ Equal contributors

${ }^{2}$ Department of Neurosurgery, University of Schleswig-Holstein Medical Center, Campus Kiel, Arnold-Heller-Str.3, Building 41, 24105 Kiel, Germany

Full list of author information is available at the end of the article
}

corresponding receptors at the surface of the same or another cell. Some tumor cells have high levels of transmembrane ligands but do not produce the corresponding receptors for these molecules. Serendipitously, in malignant brain tumor cells we detected a novel, alternate mechanism of cell communication which we term "inverse signaling": Here, a transmembrane ligand (namely transmembrane chemokines) acts as a "receptor" for its soluble counterpart. By studying now benign human tumor cells derived from the linings of the brain and spinal cord, so called meningiomas, Hattermann et al. show that the soluble form of the transmembrane 
chemokine CXCL16 binds to its transmembrane equivalent in these tumor cells, too. This interaction initiates intracellular signaling pathways that promote cell growth and make the meningioma cells more resistant to cell death. Thus, Hattermann et al. showed that the "inverse signaling" paradigm also takes place in benign tumor cells, suggesting that it helps to fine-tune the communication between cells as a broad biological process.

\section{Background}

The chemokine CXCL16 (synonym SR-PSOX) was originally discovered as a scavenger receptor for oxidized LDL [1], and independently as a ligand for the CXC-chemokine receptor CXCR6, also termed Bonzo, TYMSTR, STRL33 [2]. CXCL16 is synthesized as a transmembrane (tm) multi-domain molecule consisting of a chemokine domain followed by a glycosylated mucin-like stalk, a single transmembrane helix and an intracellular tail. However, a soluble (s-)CXCL16 form can be generated by constitutive or induced cleavage from the transmembrane form by the cell-surface proteases ADAM10 and 17 (ADAM, a disintegrin and metalloproteinase) $[3,4]$.

In previous investigations we and others determined that CXCL16 is definitely involved in tumor progression of different tumor types, e.g. gliomas, schwannomas, lung and breast tumors [5-9]. However, apart from its classical signaling mode in which the proteolytically released chemokine domain would bind to and signal via its receptor CXCR6, we recently discovered an alternate signaling mechanism for transmembrane chemokines in glioma cells which we termed "inverse signaling" [10]. According to this novel mechanism, the proteolytically released (or also recombinant) $s$-chemokine binds to an intact transmembrane chemokine specimen inducing intracellular signaling and biological effects independently from the expression of the receptor CXCR6. We initially demonstrated this mechanism in cultivated malignant human glioma cells, from which we had previously shown to express high levels of CXCL16, but a lack of the corresponding receptor CXCR6 [11].

Apart from that, we could show that solid human meningiomas - the second most common intracranial tumors - are also characterized by high expression levels of CXCL16 while CXCR6 expression is more restricted [12]. Meningiomas develop from arachnoid cap cells, and although malignant variants exist (atypical meningiomas, World Health Organization, WHO grade II and anaplastic meningiomas, WHO grade III) most meningiomas (about $90 \%$ ) are slowly growing benign (WHO grade I) tumors [13, 14].

Facing the high CXCL16 expression levels in human meningiomas, we raised the question if "inverse signaling" of CXCL16 previously observed in highly malignant glioma cell might also take place in benign meningioma cells. Therefore, we analyzed binding, intracellular signaling effects and different biological readouts in cultured primary human meningioma cells upon stimulation with $s$-CXCL16. This should validate our recent hypothesis on inverse signaling in more benign brain tumor cells as a broader biological process.

\section{Methods}

\section{Tumor specimens}

Meningioma samples were surgical dissected tissues from the Department of Neurosurgery (Kiel, Germany) and were obtained in accordance with the Helsinki Declaration of 1975 with approval of the ethics committee of the University of Kiel, Germany (file reference: D 442/ 11) after written consent of donors. Tumors were classified according to the WHO criteria into the various subtypes of meningiomas [13]. The diagnosis was established by a pathologist. In this study, a total number of 28 meningiomas were included (P1 to P28). With exception of P10, P25, P27 and P28 all meningiomas were classified into WHO grade one, P10, P25, P27 and P28 were grad two meningiomas. If possible (enough material available), for different investigations matched probes of individual tumor samples were used (see Table 1).

\section{Real-time RT-PCR (qRT-PCR)}

RNA was isolated with the TRIZOL reagent (Invitrogen, Carlsbad, CA, USA), digested by DNase, cDNA was synthesized, and quantitative real time RT-PCR (qRTPCR) was performed [12, 15] using TaqMan primer probes (Applied Biosystems, Foster City, CA, USA): hGAPDH (Hs99999905_m1), hCXCL16 (Hs00222859_m1), hCXCR6 (Hs00174843_m1). All 28 different meningioma samples were analyzed by qRT-PCR. The reaction was carried out with the $\mathrm{MyiQ}^{\text {ma }}$ Single Color Real-time PCR Detection System (BIO-RAD, München, Germany) and fluorescent data were converted into cycle threshold $\left(\mathrm{C}_{\mathrm{T}}\right)$ measurements. $\Delta \mathrm{C}_{\mathrm{T}}$ values of each sample were calculated as $\mathrm{CT}_{\text {gene of interest }}-\mathrm{CT}$ GAPDH. Relative gene expression was calculated with $2^{\text {(normalized }} \mathrm{CT}$ non-stimulated - normalized CT stimulated) $=n$-fold of control. A $\Delta C_{\mathrm{T}}$ value of 3.33 corresponds to one magnitude lower gene expression compared to GAPDH (glycerinaldehyde-3-phosphate-dehydrogenase). For each gene, logarithmic linear dependence of $\mathrm{C}_{\mathrm{T}^{-}}$ values from the numbers of copies was verified by using different amounts of cDNA.

\section{Cell culture}

It should be noted that all experiments were performed with cells cultivated from surgical dissected tumors described above. Primary human meningioma cells were cultured in glutamine-supplemented Dulbecco's modified Eagle's medium (DMEM) plus $20 \%$ fetal calf serum (FCS) as described previously [15]. Subcultures from 2 
Table 1 Documentation of patients' samples and use for experiments

\begin{tabular}{|c|c|c|c|c|c|c|}
\hline Sample & $\begin{array}{l}\text { WHO } \\
\text { grade }\end{array}$ & $\begin{array}{l}\text { Expression } \\
\text { analysis } \\
\text { qRT-PCR/ } \\
\text { ICCMB }\end{array}$ & Kinase-WB & Binding & Proliferation & Apoptosis \\
\hline P1 & I & $x$ & $x$ & & $x$ & \\
\hline P2 & I & $x$ & $x$ & & & \\
\hline P3 & I & $x$ & & & $x$ & \\
\hline P4 & I & $x$ & $x$ & & $x$ & \\
\hline P5 & I & $x$ & & & & \\
\hline P6 & I & $x$ & & $x$ & & $x$ \\
\hline P7 & I & $x$ & & & & $x$ \\
\hline P8 & I & $x$ & $x$ & & & $x$ \\
\hline P9 & I & $x$ & $x$ & & & \\
\hline P10 & $\|$ & $x$ & $x$ & & & \\
\hline P11 & I & $x$ & $x$ & & & \\
\hline P12 & I & $x$ & $x$ & & & \\
\hline P13 & I & $x$ & $x$ & & & \\
\hline P14 & 1 & $x$ & $x$ & & & \\
\hline P15 & I & $x$ & & $x$ & & \\
\hline P16 & I & $x$ & & $x$ & & \\
\hline P17 & I & $x$ & & & & \\
\hline P18 & I & $x$ & & & & \\
\hline P19 & I & $x$ & & & & \\
\hline P20 & I & $x$ & & & & \\
\hline P21 & I & $x$ & & & & \\
\hline P22 & 1 & $x$ & & & & \\
\hline P23 & I & $x$ & & & & \\
\hline P24 & I & $x$ & & & & \\
\hline P25 & $\|$ & $x$ & & & & \\
\hline P26 & I & $x$ & & & & \\
\hline P27 & $\|$ & $x$ & & & & \\
\hline P28 & $\|$ & $x$ & & & & \\
\hline
\end{tabular}

We included 24 WHO grade I and 4 WHO grade II tumor samples and used cultured meningioma cells from these samples for experiments as indicated

to 4 were used. Meningiomas express both mesenchymal and epithelial markers of which EMA (epithelial membrane antigen) and fibronectin can be detected in 90 to $100 \%$ of all investigated solid meningiomas, depending on marker and investigated cohort [16-18]. Thus, identity and purity of meningioma cell cultures were proven routinely by fibronectin (1:100; rabbit polyclonal antihuman fibronectin; Santa Cruz Biotechnology, Santa Cruz, CA), EMA (1:20; mouse monoclonal anti-human EMA; DAKO, Glostrup, Denmark), and glial fibrillary acidic protein (GFAP) (1:200; mouse monoclonal antihuman GFAP, DAKO) immunocytochemistry staining.
The primary antibody was omitted for negative controls. All cultured meningiomas showed a positive staining for EMA and fibronectin, but lack GFAP expression.

\section{Immunocytochemistry (ICC)}

For immunocytochemistry examination, cultured primary human meningioma cells were seeded on sterile glass cover slides $(50,000$ cells/well for routine and CXCL16 staining, 16,000, 64,000 and 160,000/well to investigate the density dependent expression of CXCR6) and grown for two (for density dependent expression analysis) to up to four days in $20 \%$ FCS-supplemented DMEM. In case of stimulations, cells were seeded on glass cover slides $(64,000 /$ well), grown for two days and stimulated for 24 h with $10 \mathrm{nM}$ CXCL16 (PeproTech, Hamburg, Germany). Then, cell were washed with phosphate buffered saline (PBS) for three times and fixed with methanol-acetone (1:1; ice-cold) for $10 \mathrm{~min}$ and $4 \%$ para-formaldehyde in PBS for $30 \mathrm{~min}$. Non-specific binding was blocked with $0.5 \%$ bovine serum albumin (BSA) $/ 0.5 \%$ glycine in PBS for 60 min. Glass cover slides were incubated with anti-CXCL16 or anti-CXCR6 primary antibody over night at $4{ }^{\circ} \mathrm{C}$ (anti-CXCL16, goat polyclonal, 1:100 and anti-CXCR6, mouse monoclonal, 1:100, both R\&D Systems, Wiesbaden, Germany). The primary antibody was omitted for negative controls. After washing steps with PBS, glass cover slides were incubated with the Alexa Fluor 555-coupled secondary antibody (red, 1:1,500, donkey anti-goat or anti-mouse IgG, Invitrogen, Life Technologies, Karlsruhe, Germany) for $1 \mathrm{~h}$ at $37^{\circ} \mathrm{C}$ in darkness. After washing with PBS, nuclei were stained with 4',6-diamidino-2-phenylindole (DAPI; Molecular Probes/Invitrogen; 1:30,000, $30 \mathrm{~min}$ room temperature), washed with $\mathrm{PBS}(3 \times)$ and finally distilled water. After embedding in Immu-Mount (Shandon, Pittsburgh, PA, USA) digital photography was performed using a Zeiss fluorescence microscope and Zeiss camera (Zeiss, Jena, Germany). As a positive control for CXCR6 immunoreactivity, we used LOX melanoma cells transfected with an expression vector for CXCR6 (OriGene, Rockville, MD). Native LOX melanoma cells were a kind gift of Professor Udo Schumacher, University Hospital Hamburg-Eppendorf.

\section{Binding experiments}

Cultured primary human meningioma cells were grown for up to four days on sterile glass cover slides in $20 \%$ FCS-supplemented DMEM, washed with PBS for three times and incubated for $15 \mathrm{~min}$ on ice. Meningioma cells were incubated with Cy3-labeled CXCL16 $(2 \mu \mathrm{l}$ diluted in $50 \mu \mathrm{l} \mathrm{PBS})$ or Cy3-labeled lactalbumin $(2 \mu \mathrm{l}$ diluted in $50 \mu \mathrm{l} \mathrm{PBS}$ ) for $60 \mathrm{~min}$ on ice. For labeling of CXCL16 and lactalbumin, $2 \mu \mathrm{g}$ protein were incubated with a 4-fold excess of monoreactive Cy3-NHS ester (GE 
Healthcare, Freiburg, Germany) in $0.2 \mathrm{M} \mathrm{NaHCO} 3$ buffer, $\mathrm{pH} 8.4$ (total reaction volume $90 \mu \mathrm{l}$ ). After a washing step with PBS, cells were fixed in methanolacetone $(1: 1$; ice-cold) for $10 \mathrm{~min}$, and washed for three times with PBS. Nuclei were stained with DAPI (see above), and after embedding in Immu-Mount (Shandon) digital photography was performed using a Zeiss fluorescence microscope and Zeiss camera (Zeiss).

\section{Western blot}

Primary human meningioma cells $\left(1.5 \times 10^{5}\right)$ were grown for two days in $20 \%$ FCS-supplemented DMEM, washed in DMEM with $0.5 \%$ FCS for three times $(20 \mathrm{~min}$, respectively) and stimulated for $10 \mathrm{~min}, 20 \mathrm{~min}$ and $40 \mathrm{~min}$ in the same medium with $10 \mathrm{nM}$ CXCL16 (PeproTech) or for $10 \mathrm{~min}$ with epidermal growth factor (EGF; $10 \mathrm{ng} / \mathrm{ml}$; Pepro Tech) or for $20 \mathrm{~min}$ with antiCXCL16 (1 $\mu \mathrm{g} / \mathrm{ml}$; R\&D Systems), respectively. In parallel, control cells were kept without stimulation, these were also used to confirm lack of CXCR6 expression on protein level by western blot [6]. Cells were harvested with $1 \mathrm{ml}$ lysis buffer [50 mM TRIS, $100 \mathrm{mM} \mathrm{NaCl}$, 2 mM EDTA, $1 \%$ Triton-X-100, and $1 \mathrm{mM}$ sodium vanadate, $1 \times$ Halt $^{\mathrm{TM}}$ Phosphatase Inhibitor Cocktail (Thermo Scientific, Bonn, Germany)], $3 \mu \mathrm{g}$ of protein per sample was loaded on $10 \%$ SDS-polyacrylamide gels for electrophoresis and then transferred to a polyvinylidene difluoride membrane (Hybond $^{\mathrm{TM}}-\mathrm{P}$ PVDF membrane, GE Healthcare, Freiburg, Germany). As a positive control for CXCR6, $50 \mathrm{ng} /$ lane recombinant human CXCR6 (Biozol, Eching, Germany) was applied in a separate lane. To verify CXCL16 expression in membrane isolates, and to exclude that CXCL16 stimulation might induce CXCR6 expression, meningioma cells were grown until confluency, stimulated with $10 \mathrm{nM}$ CXCL16 or not, and cells were harvested and lysed in $5 \mathrm{mH}$ Hepes, $\mathrm{pH}$ 7.4. Then, 10 vol.\% $200 \mathrm{mM}$ Hepes, $1.4 \mathrm{mM} \mathrm{NaCl} \mathrm{pH} \mathrm{7.4} \mathrm{was} \mathrm{added}$ to each sample, detritus was removed by centrifugation ( $8 \mathrm{~min}, 800 \mathrm{xg}$ ), and membranes were isolated by centrifugation for $60 \mathrm{~min}$ at $14000 \mathrm{xg}$. Membrane preparations were solubilized in $20 \mathrm{mM}$ Hepes, $0.14 \mathrm{mM} \mathrm{NaCl}, \mathrm{pH} 7.4$, and protein amounts of $5 \mu \mathrm{g} /$ lane were applied to electrophoresis and blotting as described above. The polyvinylidene difluoride membranes were blocked with $5 \%$ BSA/TBST and incubated with primary antibodies against phospho-p42/44 MAPK (Cell Signaling, Beverly, MA, 1:1,000), phospho-Akt (Cell Signaling, 1:250), CXCR6 (Acris, Hiddenhausen, Germany, 1:250) or CXCL16 (PeproTech, 1:250) at $4{ }^{\circ} \mathrm{C}$ overnight. The membranes were incubated with the secondary antibody (1:30,000, donkey anti-rabbit IgG-HRP, Santa Cruz Biotechnology) for $1 \mathrm{~h}$ at room temperature, and horseradish peroxidase activity was detected by applying an ECL
Advance Western Blotting Detection Kit (GE Healthcare) followed by exposure of the membranes to a sheet of autoradiography film (Hyperfilm ${ }^{\mathrm{TM}} \mathrm{ECL}^{\mathrm{TM}}$, GE Healthcare). Equal protein loading was confirmed by either reprobing the membranes with anti-ERK-2 (1:200, Santa Cruz Biotechnology), anti-Akt (1:500, Cell Signaling) or anti-Caveolin-1 (1:200, Santa Cruz Biotechnology) after antibody stripping for 30 min using Reblot Stripping Solution (Millipore, Temecula, CA, USA), or by performing second SDSpolyacrylamide gel with same probes in parallel.

\section{Proliferation assay}

Primary human meningioma cells were plated into 96-well dishes (1,000 cells/ well), grown for two days in $20 \%$ FCSsupplemented DMEM, and stimulated in the same medium with $10 \mathrm{nM}$ CXCL16 (Pepro Tech), $1 \mu \mathrm{g} / \mathrm{ml}$ anti-CXCL16 (R\&D Systems) or $10 \mathrm{ng} / \mathrm{ml}$ EGF for up to $24 \mathrm{~h}$. In parallel, control wells were kept without stimulation. Proliferation was determined by the measurement of tetrazolium salt WST-1 cleavage (Roche, Mannheim, Germany) and normalized to non-stimulated control ( 4 individual wells for each stimulus).

\section{Caspase-3 activity assay}

Primary human meningioma cells were plated into 96-well dishes (10,000 cells/ well), grown for two days in $20 \%$ FCS-supplemented DMEM, washed in $37{ }^{\circ} \mathrm{C}$ thermostatted $0.5 \%$ FCS-supplemented DMEM for three times (20 min, respectively), and stimulated in the same medium with $50 \mu \mathrm{g} / \mathrm{ml}$ camptothecin (Sigma Aldrich, Steinheim, Germany), 10 nM CXCL16 (Pepro Tech) or with combination of both for up to $24 \mathrm{~h}$. In parallel, control wells without/with stimulation in DMSO were used. For detection of active caspase- 3 amounts, samples were washed in PBS and incubated in $100 \mu$ l Homogeneous Caspase 3/7 substrate (Apo-ONE ${ }^{\oplus}$ Homogeneous Caspase-3/7 Assay; Promega, Madison, USA) for $30 \mathrm{~min}$ according to the manufacturer's instruction and as described before [15]. The amounts of active caspase-3 were determined in relation to a caspase 7 standard (Enzo Life Science, Lörrach, Germany), and camptothecin-stimulated wells were set $100 \%$.

\section{RNAi silencing}

After cultivation of primary human meningioma cells in DMEM plus 20 \% FCS in 6-well dishes $(150,000$ cells/well; for Western Blot experiments) or on sterile glass cover slides $(15,000$ cells/well; for binding experiments) for $24 \mathrm{~h}$, cells were transfected with siCXCL16 RNA (CXCL16 siRNA ID: s33808; 50 pmol/well; Life Technologies, Darmstadt, Germany) dissolved in a mixture of Opti-MEM Medium and lipofectamine (Life technologies) for $6 \mathrm{~h}$. In parallel a transfection with silencer select negative control siRNA (Life 
technologies) was performed under same conditions. After transfection cell culture medium was changed and meningioma cells were cultured for another $24 \mathrm{~h}$ in DMEM plus $20 \%$ FCS. Then, cells were washed 20 min for three times, respectively, with DMEM plus $0.5 \%$ FCS and afterwards stimulated for 15 min with or without recombinant CXCL16 (10 nM; PeproTech) dissolved in DMEM plus 0.5 \% FCS, lysed and applied for Western Blot experiments as described above. For binding experiments primary human meningioma cells were washed with PBS for three times, incubated for 15 min on ice, and stained with Cy3-labeled CXCL16 $(2 \mu \mathrm{l}$ diluted in $50 \mu \mathrm{l}$ PBS) or Cy3-labeled lactalbumin $(2 \mu$ ld diluted in $50 \mu \mathrm{l}$ PBS) for $60 \mathrm{~min}$ on ice. Fixation of cells and counterstaining of nuclei were performed as described above.

For controlling the knockdown efficiency, RNA of transfected cells were purified in parallel with the PicoPure RNA Isolation Kit (MDS Analytical Technologies, Sunnyvale, CA) according to the manufacturer's instructions, and qRT-PCR using TaqMan primer probes (Applied Biosystems): hGAPDH (Hs99999905_m1) and hCXCL16 (Hs00222859_m1) was performed as described above.

\section{Results \\ Cultured human meningioma cells express CXCL16, but not CXCR6}

We initially measured CXCL16 and CXCR6 mRNA (Fig. 1a) expression in cultured meningioma cells used in our experimental settings. In comparison to CXCL16, which was clearly detectable in all investigated samples, CXCR6 transcription was (nearly) undetectable. To precisely identify the primary cultures as meningioma cells, each culture was routinely immunostained for epithelial membrane antigen (EMA), fibronectin and glial fibrillary acidic protein (GFAP). Examples of this routine staining are shown in Fig. 1b. Only cultures showing positive immunostaining for EMA and fibronectin combined with negative staining for GFAP were used for experiments. On protein level, we could detect CXCL16 expression by immunocytochemistry and western blots of meningioma cell membrane preparations (Fig. 1c) yielding specific bands for the transmembrane (tm-)CXCL16. In contrast, CXCR6 could not be detected on protein level by western blot and immunocytochemistry (Fig. 1d and e), and its expression was neither provoked by stimulation with $10 \mathrm{nM}$ CXCL16 nor by cultivation of different cell densities ranging from 16,000 to 160,000 cells per well, which represent the cell densities seeded for subsequent experiments (Fig. 1e). Thus, we concluded that human meningioma cells cultivated from different patients samples substantially express CXCL16 expression while CXCR6 is absent.
Cultured primary human meningioma cells bind s-CXCL16 and show subsequently activated intracellular signaling kinases

We measured in a next step if cultured CXCR6negative human primary meningioma cells were able to bind $s$-CXCL16 and transduce signaling effects. In fact, Cy3-labeled $s$-CXCL16 was found to bind to the meningioma cells (Fig. 2a), whereas Cy3-labeled lactalbumin as a negative control did not yield any staining. Next to binding, $s$-CXCL16 was able to induce phosphorylation and thereby activation of both $\mathrm{p} 42 / 44$ extracellular mitogen-activated kinase (ERK1/2) and Akt in a time-dependent manner (Fig. 2b; exemplary results are shown). In all independent experiments, we observed an activation of the respective kinases between 10 and $40 \mathrm{~min}$. However, since primary cultures were obtained from various patients, the time course and intensity of activation differed between cultures. Stimulation with epidermal growth factor (EGF) served as a positive control.

Additionally, in accordance with our recent findings in gliomas [10], application of a specific CXCL16 antibody $(\alpha C X C L 16)$ which may also induce the intrinsic activity of $t m$-CXCL16, resulted in phosphorylation of both Akt and ERK1/2 (Fig. 2b, right side) after $20 \mathrm{~min}$.

\section{Soluble s-CXCL16 induces proliferation and rescue from apoptosis}

To understand which biological consequences were induced after s-CXCL16 induced "inverse signaling", we investigated proliferation effects and whether $s$-CXCL16 could prevent apoptosis in CXCR6-negative but tmCXCL16-positive cultured primary human meningioma cells (Fig. 3).

Indeed, after $24 \mathrm{~h}$ stimulation time with $s$-CXCL16, proliferation was induced up to $117 \%$ (P1 and P3; control $=100 \%)$ and $118 \%$ (P4; control $=100 \%$; Fig. 3a). Comparable results were detectable after application of the specific CXCL16 antibody ( $\alpha$ CXCL16). Valuating these results, one should keep in mind that cultured human meningioma cells are large and slow-growing cells. Thus, regarding the fact that the positive control yielded proliferation of meningioma cells within the same range $(\mathrm{P} 1=130 \% ; \mathrm{P} 3=132 \% ; \mathrm{P} 4=126 \%$; control $=100 \%)$, CXCL16 stimulation clearly yielded proliferative effects in these cells.

Additionally, CXCL16 was able to reduce caspase-3 activity after camptothecin treatment in CXCR6-negative but $t m$-CXCL16-positive cultured primary human meningioma cells (Fig. 3b). In detail, for sample P6 camptothecin-induced caspase-3 activity (DMSO as control $=0 \%$; camptothecin dissolved in DMSO = $100 \%$ ) was reduced up to $51 \%$, for P7 up to $65 \%$, and for P8 up to $84 \%$ after $s$-CXCL16 application. 


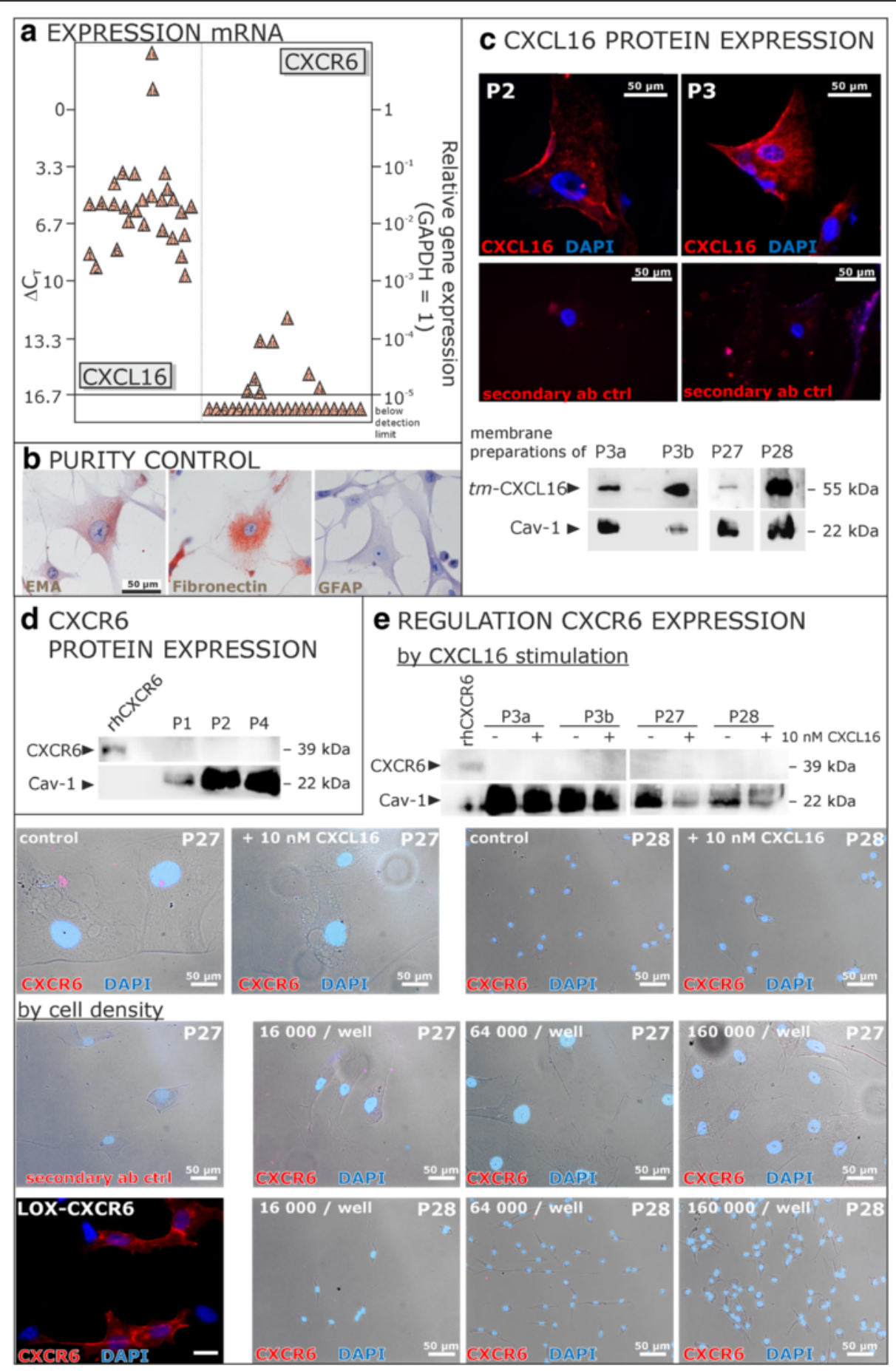

Fig. 1 (See legend on next page.) 
(See figure on previous page.)

Fig. 1 Expression of CXCL16 and its receptor CXCR6 in cells cultivated from human meningiomas. a Transcription of CXCL16 and its receptor CXCR6 in human meningioma primary cultures was determined by qRT-PCR (each triangle indicates an individual patient's sample). Meningioma primary cultures show high mRNA levels of CXCL16, whereas the expression of CXCR6 is hardly detectable or completely lost. $\mathbf{b}$ The identity and purity of meningioma cultures was assured for every culture by immunocytochemistry on EMA, fibronectin and GFAP, only cultures with positive reactivity for EMA and fibronectin and negative staining for GFAP were used for further experiments (exemplary data). c Expression of surface tm-CXCL16 on protein level was confirmed by immunocytochemistry using a CXCL16 specific antibody and a red labeled secondary antibody, nuclei were counterstained with DAPI. Furthermore, membrane expression of CXCL16 was proven by western blot using membrane preparations isolated from meningioma cells. Caveolin-1 (Cav-1) served as membrane-specific loading control. $\mathbf{d}$ Lack of CXCR6 expression was confirmed on protein level by western blot. In parallel to subsequent experiments, cell lysates were analyzed using a CXCR6-specific antibody. Whereas the positive control, human recombinant CXCR6, was sensitively detected (50 ng/lane), cultured meningioma cells did not show any CXCR6 expression. e CXCR6 expression was neither induced by CXCL16 stimulation nor by different cell densities. Stimulation with $10 \mathrm{nM}$ recombinant human CXCL16 for $24 \mathrm{~h}$, the maximum stimulation time for subsequent experiments, did not yield an induction of CXCR6 expression as shown by ICC and western blot. Additionally, different cell densities in a range from 16,000 to 160,000 cell/well (representing the cell densities seeded for subsequent experiments) did not influence on the expression of CXCR6 as shown by ICC. Exemplary data of technical (P3 a and $\mathbf{b}$ different cultivation passages) and biological replicates are shown. LOX melanoma cells which were transfected with an expression vector for human CXCR6 served as a positive control to confirm the specificity of the CXCR6 antibody in ICC, recombinant human CXCR6 served as positive control for western blot experiments

\section{CXCL16 knockdown showed specificity of results}

In a next step, we aimed to show that tm-CXCL16 expression is mandatory to the signaling mediated by $s$-CXCL16, to further support the hypothesis of "inverse signaling" in human meningioma cells. In our recent investigations in cultured human glioma cells

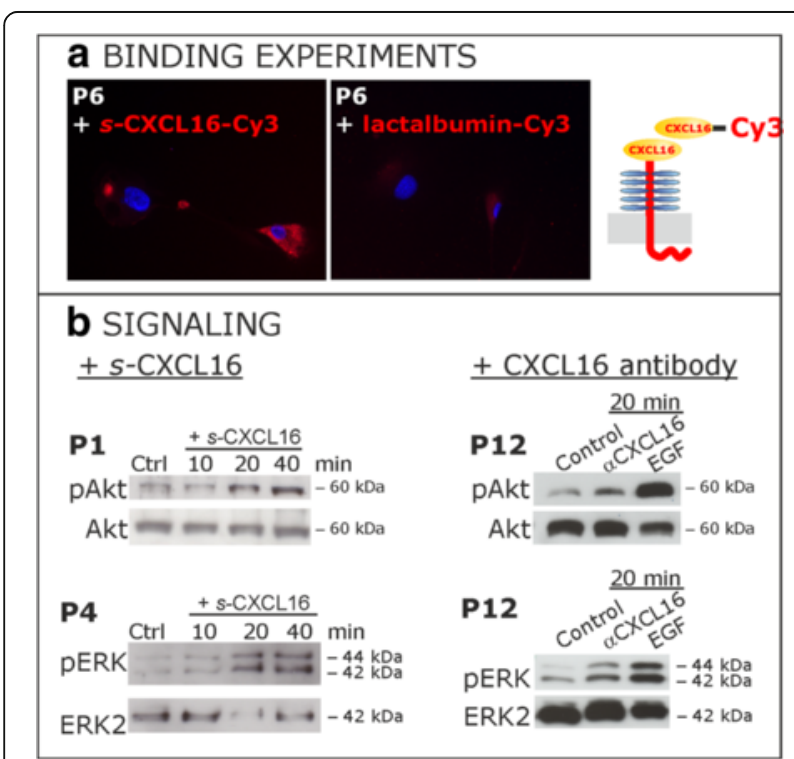

Fig. 2 CXCL16 binds to meningioma cells and activates intracellular signaling pathways. a Cy3-labeled s-CXCL16 binds to primary human meningioma cells, while after incubation with Cy3-labeled lactalbumin binding could not be observed (equal exposure times, representative examples). $\mathbf{b}$ Stimulation of cultured primary meningioma cells with $10 \mathrm{nM}$ s-CXCL16 (left) yields a time-dependent activation of the signal kinases ERK1/2 and Akt as detected by western blot using antibodies for the phosphorylated kinases (pAkt and pERK). Equal protein loading was confirmed by detection of the non-phosphorylated kinases (Akt and ERK2), respectively. This effect was also observed when a CXCL16targeting antibody (right, aCXCL16, $1 \mu \mathrm{g} / \mathrm{ml}$ ) was used for stimulations. EGF served as positive control, shown are representative examples of at least 3 independent experiments with cultures from different donors we proved the specificity of this effect by several different approaches [10]. Due to limited material and characteristics of cultured meningioma cells (e.g. decreased proliferation and increased susceptibility to apoptosis after transfection) we chose binding experiments and kinase activation as model experiments to prove the specificity of the results by CXCL16 knockdown. Results are shown in Fig. 4.

Binding of Cy3-labeled s-CXCL16 to tm-CXCL16 expressed by cultured human meningioma cells was almost completely abolished after siCXCL16 knockdown in the tumor cells (Fig. 4a, upper part). In contrast, control siRNA transfected meningioma cells were still able to bind $s$-CXCL16. Cy3-labeled lactalbumin served as a negative control for testing unspecific binding background. The expression of CXCL16 as determined by qRT-PCR was reduced to $6.7 \%$ (P15) and to $46 \%$ (P16; Fig. 4b).

Additionally, in relation to meningioma cells transfected with control siRNA, siCXCL16 transfected ones showed reduced phosphorylation and thereby activation of ERK1/2 after $s$-CXCL16 treatment (exemplary results in Fig. 4a, lower part). These results are sustained by the reduction of CXCL16 mRNA expression to $11.5 \%$ (P9) and $28.7 \%$ (P10; Fig. 4b, bottom; control siRNA = $100 \%$ ) as determined by qRT-PCR.

Summarized, in cultured primary human meningioma cells the classical CXCL16 receptor CXCR6 is lost, but nevertheless CXCL16 is still able to bind to and transduce signals into the cells resulting in increased proliferation and rescue of apoptosis of the cells. The binding and subsequent cellular activation clearly depends on the expression of tm-CXCL16 as shown by siRNA knockdown. Thus, "inverse signaling" of the transmembrane chemokine CXCL16 occurs in cultured primary human meningioma cells, and is involved in progression of human meningiomas. 


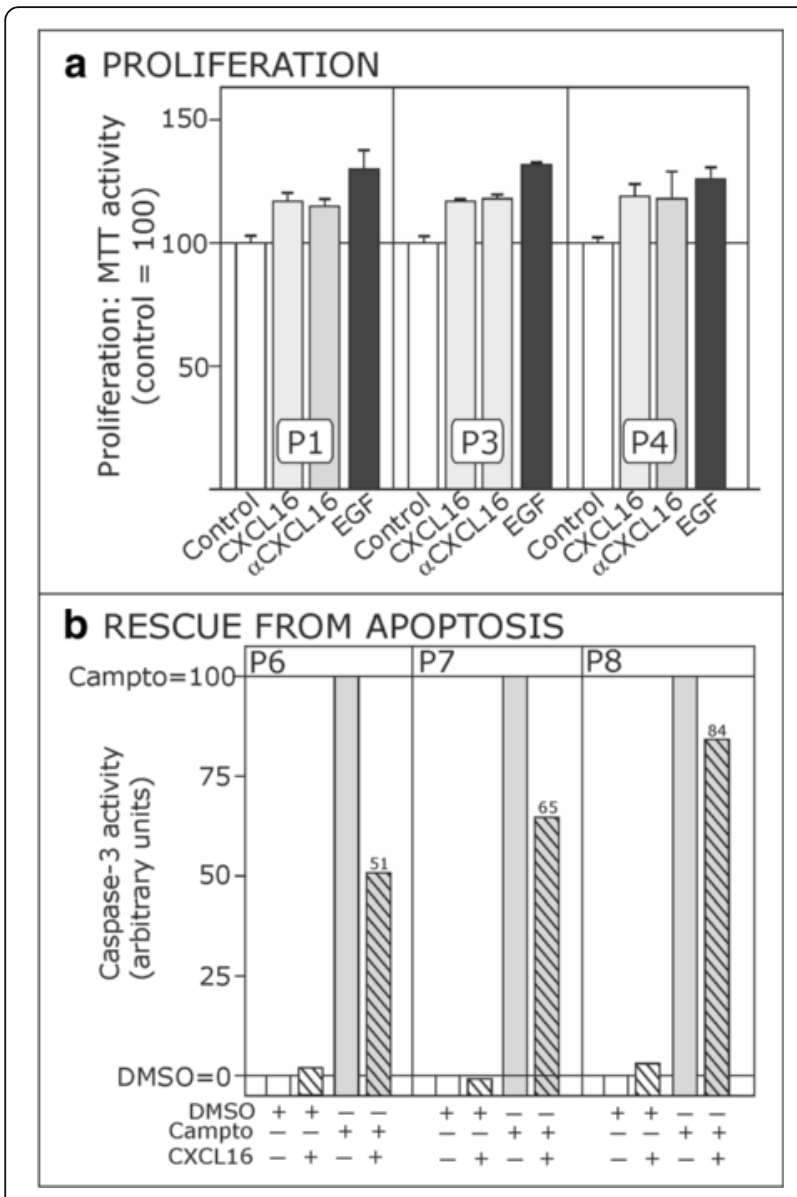

Fig. $3 \mathrm{~s}$-CXCL16-mediated effects on proliferation and rescue from apoptosis. a $10 \mathrm{nM} \mathrm{s-CXCL16}$ and $1 \mu \mathrm{g} / \mathrm{ml}$ aCXCL16 promote the proliferation of slowly growing human meningioma cells as measured by MTT activity assay. EGF $(10 \mathrm{ng} / \mathrm{ml})$ served as positive control. Control values were set $100 \%$, and shown are three independent experiments with cultures from different donors, with mean \pm SD from 4 technical replicates. b When apoptosis was induced in human primary meningioma cells by $50 \mathrm{\mu g} / \mathrm{ml}$ camptothecin (Campto $=100 \%$ ), additional incubation with $10 \mathrm{nM} \mathrm{CXCL16}$ could drastically reduce the caspase- 3 activity. Shown are three independent experiments with cultures from different donors

\section{Discussion}

We recently discovered in highly malignant glioma cells a novel form of para- or autocrine signaling mechanism for transmembrane chemokines which we termed "inverse signaling" [10]. This new signaling concept anticipates that the proteolytically released chemokine domain ( $s$-chemokine) specifically binds to its transmembrane counterpart resulting in activation of intracellular kinases and induction of proliferation and anti-apoptosis. Thus, in this novel signaling concept, the transmembrane ligands act as receptors for their soluble counterparts. This signaling concept war sustained by various silencing and transfection experiments. It requires di-/ multimerization of the $t m$-chemokine, which can also be induced by di-, but not by monovalent (Fab-fragments) antibodies. We could also show that the intracellular domain (that contains motifs for binding adapter proteins) is essential for signaling. In the present investigation we now describe that cultured primary human meningioma cells exhibited high CXCL16 expression in vitro, whereas the CXCL16-specific chemokine receptor CXCR6 was mostly absent. Facing the discrepancy between tm-chemokine expression and the lack of the corresponding receptor also in meningioma cells, we hypothesized that the concept of inverse signaling as a broad biological concept may be extended further to more benign tumor entities.

The expression of the chemokine CXCL16 has been reported previously for monocytes/macrophages, B cells, dendritic cells, keratinocytes and endothelial cells $[1,5,19,20]$ while the receptor CXCR6 has been detected quite selectively on activated $\mathrm{T}$ cells, NK cells and bone marrow plasma cells [2, 20, 21]. Apart from its physiological expression, CXCL16 is pathologically expressed in cancer cells of different origin including malignant glioma cells and reactive astrocytes in situ and in vitro [5-7, 11, 22].

However, although cultured human meningioma cells lack the CXCL16-specific receptor CXCR6, stimulation with $s$-CXCL16 was able to transduce intracellular signaling effects. The recombinant $s$-CXCL16 was able to bind to the cell surface and to induce phosphorylation and thereby activation of the kinases ERK and Akt in a time dependent manner. These results are in a way comparable with previous ones which described CXCL16mediated ERK activation in schwannoma cells [6, 23], or Akt activation in human aortic smooth muscle cells [24]. However, as the human cultured meningioma cells lack the corresponding receptor CXCR6, a classical receptor mediated signaling could be excluded. To investigate the relevance of $t m$-CXCL16 in the observed signaling process, we performed siCXCL16 knockdown experiments and were able to show that binding to and induction of intracellular signals clearly depends on the expression of $t m$-CXCL16.

In general, the activation of the ERK signal transduction pathway often results in elevated cell growth, and the Akt pathway in anti-apoptotic mechanisms of tumor cells. Therefore, we wanted to know whether stimulation with $s$-CXCL16 could induce these biological responses in cultured primary human meningioma cells. In fact, we could show that both effects - activation of proliferation and rescue of apoptosis - were triggered by $s$-CXCL16 in cultured CXCR6-negative, but $t m$-CXCL16-positive meningioma cells. Additionally, for a more general view on the biological role and regulation of the "inverse signaling" of CXCL16 one should keep in mind that tm-chemokines 


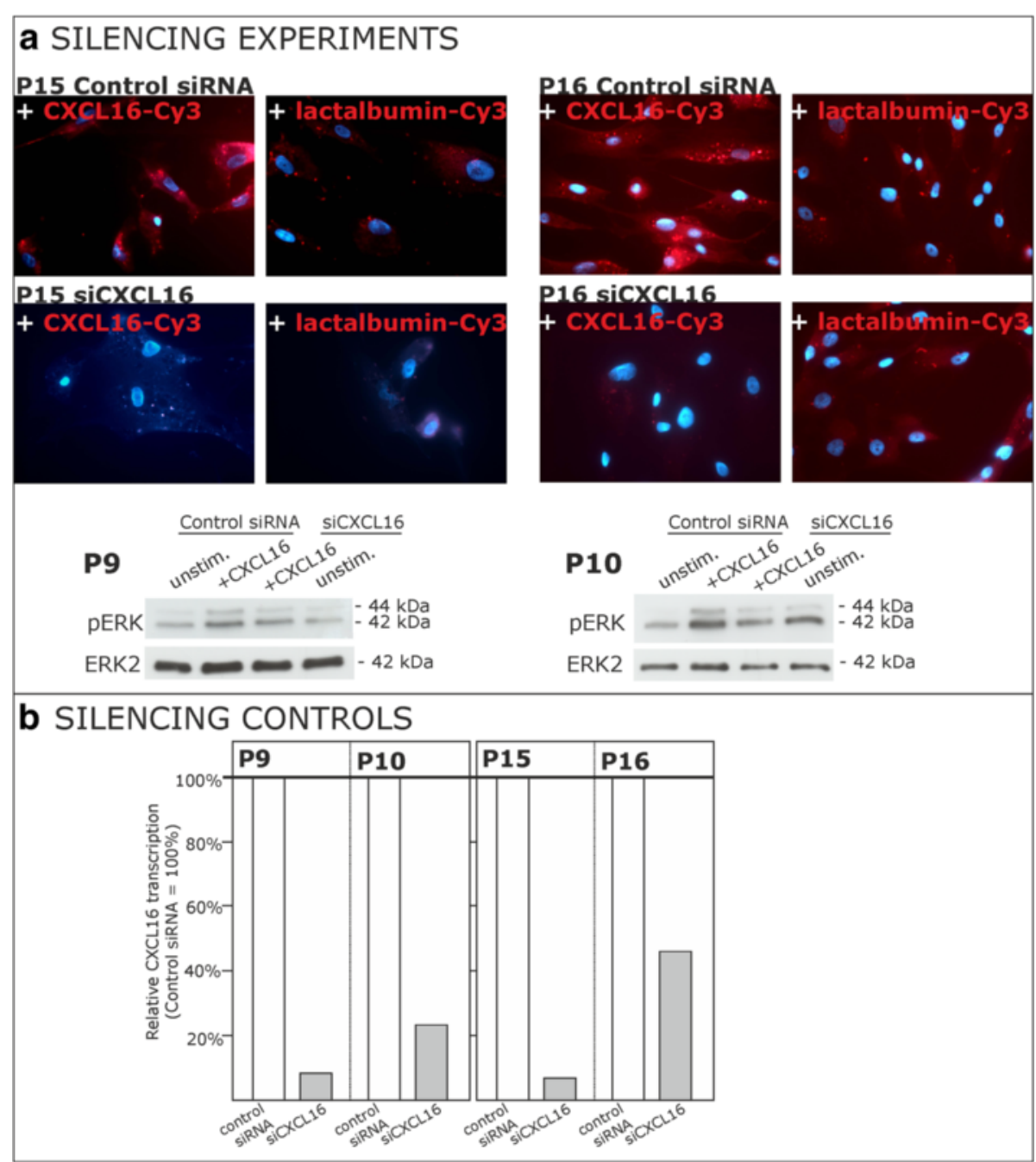

Fig. 4 Binding of s-CXCL16 and signaling upon stimulation with s-CXCL16 depends on the tm-CXCL16 level. Expression of the tm-CXCL16 was reduced in human meningioma cells by siRNA targeting CXCL16 (for controls, an unspecific control siRNA was used). a Knockdown of CXCL16 yielded a drastically reduced binding signal of Cy3-labeled s-CXCL16 (upper part). Additionally, we observed reduced activation of the ERK signaling pathway as determined by Western blot on kinase phosphorylation (lower part). $\mathbf{b}$ The efficient knockdown of CXCL16 was determined by qRT-PCR (bottom right). Shown are 2 independent experiments with meningioma primary cultures from different donors

like CXCL16 need to be cleaved by the cell surface proteases ADAM10 and ADAM17 in order to produce their soluble counterparts [5]. Regarding the biological role of CXCL16 in tumors, contrasting its already mentioned effects in glioma and schwannoma cells [5, 6, 21], high CXCL16 expression correlates with good prognosis and increased levels of tumorinfiltrating lymphocytes in renal cancer [25], while in human rectal cancer a downregulation of CXCL16 has been reported [26]. However, pro-tumorigenic effects of CXCL16 have been reported for several other tumors, e.g. breast cancer $[9,27]$, lung cancer, and is discussed as a promotor of inflammation-associated cancer in general [28], which is underlined by our own findings, that microglia/macrophages isolated from human glioma samples show high expression levels of CXCL16 themselves [29]. Thus, the inverse signaling mechanism of CXCL16 we here describe for meningiomas may occur in a variety of benign and malignant tumors, modulating amongst others the interplay between tumor and immune cells and influencing on tumor progression. Targeting the tumor promoting effects of CXCL16 might be a promising therapy amendment e.g. for atypic meningiomas and other tumors with limited treatment options.

\section{Conclusion}

Summarized, in the present investigations we were able to show that "inverse signaling" takes place in cultured 
primary human meningioma cells resulting in increased proliferation and rescue of apoptosis of these cells. Thereby, we verified this new mechanism beside its appearance in malignant glioma cells [10] in a different system, and showed that "inverse signaling" seems to be a broad biological process as it is also observable in more benign tumor cells.

\section{Abbreviations}

ADAM: A disintegrin and metalloproteinase; $C_{T}$ : Cycle of threshold; DMEM: Dulbecco's modified Eagle's medium; EGF: Epidermal growth factor; EMA: Epithelial membrane antigen; FCS: Fetal calf serum; GAPDH: Glycerinaldehyde-3-phosphate-dehydrogenase; GFAP: Glial fibrillary acidic protein; ICC: Immunocytochemistry; qRT-PCR: Quantitative reverse-transcription polymerase chain reaction; s: Soluble; tm: Transmembrane; WHO: World Health Organization

\section{Acknowledgements}

We thank Brigitte Rehmke, Fereshteh Ebrahim, Jörg Krause and Martina Burmester for expert technical assistance.

\section{Funding}

This work was supported by the University of Kiel, the "Deutsche Forschungsgemeinschaft" (HE 3400/5-1; ME 758/10-1), and by the popgen 2.0 network [(P2N; supported by a grant from the German Ministry for Education and Research (01EY1103)]. The funding bodies did not have any influence on the design of the study, the collection, analysis and interpretation of data or in the writing of the manuscript.

\section{Availability of data and material}

The datasets generated and/or analysed during the current study are available from the corresponding author on reasonable request.

\section{Authors' contribution}

$\mathrm{KH}, \mathrm{JHF}, \mathrm{RM}$ : Conception and design of the study, interpretation of data, writing of the manuscript. KB, HG: Performance of experiments, data analysis. ADS, HMM, MS: Acquisition of patients' material and data. All authors have significantly contributed to the content of the manuscript including carefully and critically revising and approved the final manuscript version.

\section{Competing interests}

The authors declare that they have no competing interests.

\section{Consent for publication}

Not applicable.

\section{Ethics approval and consent to participate}

All human materials were obtained in accordance with the Helsinki Declaration of 1975 with approval of the ethics committee of the University of Kiel, Germany (file reference: D 442/11) after written consent of donors.

\section{Author details \\ 'Department of Anatomy, University of Kiel, Otto-Hahn-Place 8, 24118 Kiel, Germany. ${ }^{2}$ Department of Neurosurgery, University of Schleswig-Holstein Medical Center, Campus Kiel, Arnold-Heller-Str.3, Building 41, 24105 Kiel, Germany.}

Received: 3 August 2016 Accepted: 14 October 2016

\section{Published online: 27 October 2016}

\section{References}

1. Shimaoka T, Kume N, Minami M, Hayashida K, Kataoka H, Kita T, Yonehara S. Molecular cloning of a novel scavenger receptor for oxidized low density lipoprotein, SR-PSOX, on macrophages. J Biol Chem. 2000;275:40663-6.

2. Matloubian M, David A, Engel S, Ryan JE, Cyster JG. A transmembrane CXC chemokine is a ligand for HIV-coreceptor Bonzo. Nat Immunol. 2000;1:298-304.

3. Abel S, Hundhausen C, Mentlein R, Schulte A, Berkhout TA, Broadway N, et al. The transmembrane CXC-chemokine ligand 16 is induced by IFN-gamma and TNF-alpha and shed by the activity of the disintegrin-like metalloproteinase ADAM10. J Immunol. 2004;172:6362-72.

4. Gough PJ, Garton KJ, Wille PT, Rychlewski M, Dempsey PJ, Raines EW. A disintegrin and metalloproteinase 10-mediated cleavage and shedding regulates the cell surface expression of CXC chemokine ligand 16. I Immunol. 2004:172:3678-85

5. Ludwig A, Schulte A, Schnack C, Hundhausen C, Reiss K, Brodway N, Held-Feindt J, Mentlein R. Enhanced expression and shedding of the transmembrane chemokine CXCL16 by reactive astrocytes and glioma cells. J Neurochem. 2005;93:1293-303.

6. Held-Feindt J, Rehmke B, Mentlein R, Hattermann K, Knerlich F, Hugo H-H et al. Overexpression of CXCL16 and its receptor CXCR6/Bonzo promotes growth of human schwannomas. Glia. 2008;56:764-74.

7. Hattermann K, Ludwig A, Gieselmann V, Held-Feindt J, Mentlein R. The chemokine CXCL16 induces migration and invasion of glial precursor cells via its receptor CXCR6 / Bonzo. Mol Cell Neurosci. 2008;39:133-41.

8. Hu W, Liu Y, Zhou W, Si L, Ren L. CXCL16 and CXCR6 are coexpressed in human lung cancer in vivo and mediate the invasion of lung cancer cell lines in vitro. PLoS One. 2014:9:e99056.

9. Fang Y, Henderson FC Jr, Yi Q, Lei Q, Li Y, Chen N. Chemokine CXCL16 expression suppresses migration and invasiveness and induces apoptosis in breast cancer cells. Mediators Inflamm. 2014;2014:478641.

10. Hattermann K, Gebhardt H, Krossa S, Ludwig A, Lucius R, Held-Feindt J, Mentlein R. Transmembrane chemokines act as receptors in a novel mechanism termed inverse signaling. eLIFE. 2016;5:e10820.

11. Hattermann K, Held-Feindt J, Ludwig A, Mentlein R. The CXCL16-CXCR6 axis in glial tumors. J Neuroimmunol. 2013;260:47-54.

12. Li G, Hattermann K, Mentlein R, Mehdorn HM, Held-Feindt J. The transmembrane chemokines CXCL16 and CX3CL1 and their receptors are expressed in human meningiomas. Oncol Rep. 2013;29:563-70.

13. Louis DN, Scheithauer BW, Budka H, von Deimling A, Kepes JJ. Meningiomas, in World Health Organization Classification of Tumours: Pathology and Genetics of Tumours of the Nervous System. In: Kleihues P and Cavenee WK, editors. Lyon: IARC Press; 2000. p. 176-184.

14. Whittle IR, Smith C, Navoo P, Collie D. Meningiomas. Lancet. 2004;363:1535-43.

15. Tong $Y$, Mentlein $\mathrm{R}$, Buhl $\mathrm{R}$, Hugo H-H, Mehdorn HM, Held-Feindt J. Overexpression of midkine contributes to anti-apoptotic effects in human meningiomas. J Neurochem. 2007;100:1097-107.

16. Ng HK, Wong AT. Expression of epithelial and extracellular matrix protein markers in meningiomas. Histopathology. 1993;22:113-25.

17. Das A, Tan WL, Smith DR. Expression of extracellular matrix markers in benign meningiomas. Neuropathology. 2003;23:275-81.

18. Hitchcock E. Morris CS Immunocytochemistry of intracranial meningiomas. J Neurooncol. 1987:5:357-68.

19. van der Voort R, van Lieshout AW, Toonen LW, Sloetjes AW, van den Berg WB, Figdor CG, et al. Elevated CXCL16 expression by synovial macrophages recruits memory T cells into rheumatoid joints. Arthritis Rheum. 2005;52:1381-91.

20. Hase K, Murakami T, Takatsu H, Shimaoka T, limura M, Hamura K, et al. The membrane-bound chemokine CXCL16 expressed on follicle-associated epithelium and M cells mediates lympho-epithelial interaction in GALT. J Immunol. 2006;176:43-51.

21. Kim CH, Kunkel EJ, Boisvert J, Johnston B, Campbell JJ, Genovese MC, et al. Bonzo/CXCR6 expression defines type 1-polarized T-cell subsets with extralymphoid tissue homing potential. J Clin Invest. 2001;107:595-601.

22. Ludwig A, Mentlein R. Glial cross-talk by transmembrane chemokines CX3CL1 and CXCL16. J Neuroimmunol. 2008;198:92-7.

23. Rangwala R, Banine F, Borg J-P, Sherman LS. Erbin regulates mitogen-activated protein (MAP) kinase activation and MAP kinase-dependent interactions between merlin and adherens junction complexes in Schwann cells. J Biol Chem. 2005;280:11700-97.

24. Chandrasekar B, Bysani S, Mummidi S. CXCL16 signals via Gi, phosphatidylinositol 3-kinase, Akt, lkB kinase, and nuclear factor-kB and induces cell-cell adhesion and aortic smooth muscle cell proliferation. J Biol Chem. 2004;279:3188-96.

25. Gutwein P, Schramme A, Sinke N, Abdel-Bakky MS, Voss B, Obermüller N, et al. Tumoural CXCL16 expression is a novel prognostic marker of longer survival times in renal cell cancer patients. Eur J Cancer. 2009:45:478-89.

26. Wågsäter D, Hugander A, Dimberg J. Expression of CXCL16 in human rectal cancer. Int J Mol Med. 2004;14:65-9.

27. Xiao G, Wang X, Wang J, Zu L, Cheng G, Hao M, Sun X, Xue Y, Lu J, Wang J. CXCL16/CXCR6 chemokine signaling mediates breast cancer progression by pERK1/2-dependent mechanisms. Oncotarget. 2015;6:14165-78. 
28. Darash-Yahana M, Gillespie JW, Hewitt SM, Chen YY, Maeda S, Stein I, Singh SP, Bedolla RB, Peled A, Troyer DA, Pikarsky E, Karin M, Farber JM. The chemokine CXCL16 and its receptor, CXCR6, as markers and promoters of inflammationassociated cancers. PLoS One. 2009:4:e6695.

29. Hattermann K, Sebens S, Helm O, Schmitt AD, Mentlein R, Mehdorn HM, Held-Feindt J. Chemokine expression profile of freshly isolated human glioblastoma-associated macrophages/microglia. Oncol Rep. 2014;32:270-6.

Submit your next manuscript to BioMed Central and we will help you at every step:

- We accept pre-submission inquiries

- Our selector tool helps you to find the most relevant journal

- We provide round the clock customer support

- Convenient online submission

- Thorough peer review

- Inclusion in PubMed and all major indexing services

- Maximum visibility for your research

Submit your manuscript at www.biomedcentral.com/submit
Biomed Central 